\title{
A relativistic calculation of the deuteron threshold electrodisintegration at backward angles
}

\author{
A. Arriaga \\ Centro de Fúsica Nuclear da Universidade de Lisboa, 1649-003 Lisboa \\ Departamento de Física, Faculdade de Ciências \\ da Universidade de Lisboa, 1700 Lisboa, Portugal \\ R. Schiavilla \\ Jefferson Lab, Newport News, VA 23606 \\ Department of Physics, Old Dominion University, Norfolk, VA 23529, USA
}

(Dated: April 13, 2007) 


\begin{abstract}
The threshold electrodisintegration of the deuteron at backward angles is studied with a relativistic Hamiltonian, including a relativistic one-pion-exchange potential (OPEP) with off-shell terms as predicted by pseudovector coupling of pions to nucleons. The bound and scattering states are obtained in the center-of-mass frame, and then boosted from it to the Breit frame, where the evaluation of the relevant matrix elements of the electromagnetic current operator is carried out. The latter includes, in addition to one-body, also two-body terms due to pion exchange, as obtained, consistently with the OPEP, in pseudovector pion-nucleon coupling theory. The full Lorentz structure of these currents is retained. In order to estimate the magnitude of the relativistic effects we perform, for comparison, the calculation with a non-relativistic phase-equivalent Hamiltonian and the standard non-relativistic expressions for the one-body and two-body pion-exchange currents. Our results for the electrodisintegration cross section show that, in the calculations using one-body currents, relativistic corrections become significant (i.e., larger than 10\%) only at high momentum transfer $Q$ ( $Q^{2} \simeq 40 \mathrm{fm}^{-2}$ and beyond). However, the inclusion of two-body currents makes the relativistic predictions considerably smaller than the corresponding non-relativistic results in the $Q^{2}$ region (18-40) $\mathrm{fm}^{-2}$. The calculations based on the relativistic model also confirm the inadequacy, already established in a non-relativistic context, of the present electromagnetic current model to reproduce accurately the experimental data at intermediate values of momentum transfers.
\end{abstract}

PACS numbers: 24.10.Jv,25.10.+s,25.30.Fj 


\section{INTRODUCTION}

The problem of how to treat the relativistic dynamics of interacting, composite objects, such as nucleons, is highly non trivial, and a variety of different approaches have been developed. These fall essentially into two classes: either field-theory inspired methods, such as, for example, the spectator [1] and Blankenbecler-Sugar [2] covariant reductions of the Bethe-Salpeter equation, or methods based on relativistic Hamiltonian dynamics (for a review, see Ref. [3]). The former include explicitly anti-particle degrees of freedom and are manifestly covariant, while the latter subsume these degrees of freedom into effective potentials, only retain particle (i.e, positive energy) propagation in the intermediate states, and typically satisfy the requirements of relativistic covariance only approximately - these and additional issues are discussed in considerable detail in a review by Gilman and Gross [4].

Both these methods - field-theory inspired and relativistic Hamiltonian dynamics - have been used in calculations of few-nucleon properties, including binding energies, momentum distributions, and electromagnetic form factors. Among the many references (a large, but non-exhaustive, listing of them is in Ref. [4]), we only mention here the calculations of: deuteron form factors [5] and triton binding energy [6] in the spectator-equation formalism; deuteron form factors within the framework of relativistic Hamiltonian dynamics, in the front-form [7], point-form [8], and instant-form [9] implementations of it; binding energies and momentum distributions of $A=3$ and 4 nuclei in instant-form Hamiltonian dynamics [10, 11].

In the present work we study the deuteron threshold electrodisintegration at backward angles with a relativistic Hamiltonian, including a relativistic one-pion-exchange potential with off-energy-shell terms as predicted by pseudovector coupling of pions to nucleons. The electromagnetic current is taken to consist of one- and two-body terms, the latter too derived from pseudovector pion-nucleon interactions. The full Lorentz structure of these currents is retained in the calculation of their matrix elements between the initial deuteron and final $n p$ continuum states. Corrections associated with the boosting of these states from the center-of-mass to the Breit frame, in which the evaluation of the matrix elements is actually carried out, are also taken into account.

The deuteron threshold electrodisintegration proceeds predominantly via a magneticdipole transition between the bound deuteron and ${ }^{1} \mathrm{~S}_{0}$ scattering state. Since the early 
seventies, it has been known [12] that the associated (isovector) transition form factor is dominated, at momentum transfers of $\simeq(8-16) \mathrm{fm}^{-2}$, by the contributions of two-body currents of pion range. To the best of our knowledge, all calculations of the cross section for this process have been carried out so far within essentially a non-relativistic framework (see, for example, Refs. $[13,14])$. One exception we are aware of is the front-form Hamiltonian dynamics study of Ref. [15], which, however, only included single-nucleon currents.

One of the goals of the present work is to assess the importance of relativistic effects in the deuteron threshold electrodisintegration. To this end, we also perform the calculation of the cross section with a non-relativistic Hamiltonian, phase-equivalent to the relativistic Hamiltonian described above, and the (standard) non-relativistic limits of the one-body and two-body pion-exchange current operators.

This paper is organized into five sections. In Sec. II we discuss the relativistic Hamiltonian from which the bound and scattering states are obtained, and the method used to boost these states from the center-of-mass to an arbitrary frame. In Sec. III we list the relativistic expressions adopted for the one-body and two-body pion-exchange currents, while in Sec. IV we illustrate the momentum-space evaluation of the relevant matrix elements entering the cross section of the deuteron threshold electrodisintegration. Finally, in Sec. V we present the results along with a discussion and concluding remarks. Details of the calculation are relegated in the Appendices.

\section{THE $n p$ BOUND AND SCATTERING WAVE FUNCTIONS}

The relativistic Hamiltonian used to generate the bound and scattering wave functions in the $n p$ rest frame is taken to be $[10,11,16]$

$$
H^{\mu}=2 \sqrt{p^{2}+m^{2}}+v^{\mu}
$$

where $v^{\mu}$ consists of a short-range part $v_{R}$ parameterized as in the Argonne $v_{18}$ potential [17], and of a relativistic one-pion-exchange potential (OPEP) given by

$$
\begin{aligned}
v_{\pi}^{\mu}\left(\mathbf{p}^{\prime}, \mathbf{p}\right)= & -\frac{f_{\pi N N}^{2}}{m_{\pi}^{2}} \frac{m}{E^{\prime}} \frac{f_{\pi}^{2}(k)}{m_{\pi}^{2}+k^{2}} \frac{m}{E}\left[\boldsymbol{\sigma}_{1} \cdot \mathbf{k} \boldsymbol{\sigma}_{2} \cdot \mathbf{k}\right. \\
& \left.+\mu\left(E^{\prime}-E\right)\left(\frac{\boldsymbol{\sigma}_{1} \cdot \mathbf{p}^{\prime} \boldsymbol{\sigma}_{2} \cdot \mathbf{p}^{\prime}}{E^{\prime}+m}-\frac{\boldsymbol{\sigma}_{1} \cdot \mathbf{p} \boldsymbol{\sigma}_{2} \cdot \mathbf{p}}{E+m}\right)\right] \boldsymbol{\tau}_{1} \cdot \boldsymbol{\tau}_{2} .
\end{aligned}
$$


Here $m$ denotes the nucleon mass, $f_{\pi N N}$ is the pion-nucleon coupling constant $\left(f_{\pi N N}^{2} / 4 \pi\right.$ $=0.075), \mathbf{p}$ and $\mathbf{p}^{\prime}$ are the initial and final relative momenta in the center-of-mass frame, $E=\sqrt{p^{2}+m^{2}}$ and $E^{\prime}=\sqrt{p^{\prime 2}+m^{2}}$ are the corresponding energies, and $\mathbf{k}=\mathbf{p}-\mathbf{p}^{\prime}$ is the momentum transfer. The monopole form factor $f_{\pi}(k)=\left(\Lambda_{\pi}^{2}-m_{\pi}^{2}\right) /\left(\Lambda_{\pi}^{2}+k^{2}\right)$ with $\Lambda_{\pi}=1.2$ $\mathrm{GeV} / \mathrm{c}$ is considered in the present work.

The $\mu$-dependent term characterizes possible off-energy-shell extensions of OPEP and, in particular, the value $\mu=1(\mu=-1)$ is predicted by pseudovector (pseudoscalar) coupling of pions to nucleons, while $\mu=0$ corresponds to the so-called "minimal non-locality" choice [18]. As shown by Friar almost three decades ago [18], these various off-shell extensions of OPEP are related to each other by a unitary transformation, that is

$$
H^{\mu}=\mathrm{e}^{-\mathrm{i} \mu U} H^{\mu=0} \mathrm{e}^{\mathrm{i} \mu U} \simeq H^{\mu=0}+\mathrm{i} \mu\left[H^{\mu=0}, U\right]
$$

if terms of $2 \pi$-range (and shorter-range) are neglected. The hermitian operator $U$ is given explicitly in Ref. [16]. This unitary equivalence implies that predictions for electromagnetic observables, such as the deuteron electrodisintegration cross section under consideration here, are independent of the particular off-shell extension adopted for OPEP, provided that the electromagnetic current operator, specifically its two-body components associated with pion exchange, is derived consistently with this off-shell extension. As discussed later in Sec. III, the pion-exchange two-body currents used in this work have been obtained assuming pseudovector coupling, and therefore the $\mu=1$ prescription is taken for OPEP. From now on, the $\mu=1$ superscript is dropped from $H^{\mu}$ in Eq. (2.1) for simplicity. The resulting relativistic Hamiltonian has been constructed to be phase-equivalent to the non-relativistic $H$, based on the Argonne $v_{18}$ potential.

The momentum-space wave functions of the deuteron and $n p$ scattering states are denoted respectively as $\psi_{M}(\mathbf{p} ; 0)$ and $\psi_{\mathbf{k} ; S M_{S}, T}^{(-)}(\mathbf{p} ; 0)$, where $\mathbf{p}$ is the relative momentum and the zero in the argument indicates the rest frame in which the deuteron and $n p$ pair have velocity $\mathbf{V}=0$. The bound-state wave function with spin projection $M$ is written as in Ref. [9], whereas the wave function corresponding to a scattering state with the $n p$ pair having relative momentum $\mathbf{k}$, and spin, spin projection, and isospin $S, M_{S}$, and $T\left(M_{T}=0\right.$ for $\left.n p\right)$, respectively, is obtained from solving the Lippmann-Schwinger equation in momentum space:

$$
\psi_{\mathbf{k} ; S M_{S}, T}^{(-)}(\mathbf{p} ; 0)=\phi_{\mathbf{k} ; S M_{S}, T}(\mathbf{p} ; 0)
$$




$$
+\sum_{M_{S}^{\prime}} \int \frac{d \mathbf{k}^{\prime}}{(2 \pi)^{3}} \frac{1}{2} \frac{1}{E_{k}-E_{k^{\prime}}-i \epsilon}\left[T_{M_{S}, M_{S}^{\prime}}^{S T}\left(\mathbf{k}, \mathbf{k}^{\prime}\right)\right]^{*} \phi_{\mathbf{k}^{\prime} ; S M_{S}^{\prime}, T}(\mathbf{p} ; 0)
$$

where the $\phi$ 's are antisymmetric two-nucleon free states - hence the factor $1 / 2$ in the integral over intermediate states $\mathbf{k}^{\prime}$ - with

$$
\phi_{\mathbf{k} ; S M_{S}, T}(\mathbf{p} ; 0)=\frac{(2 \pi)^{3}}{\sqrt{2}}\left[\delta(\mathbf{k}-\mathbf{p})-(-)^{S+T} \delta(\mathbf{k}+\mathbf{p})\right] \chi_{M_{S}, 0}^{S, T}
$$

and $E_{k}=2 \sqrt{k^{2}+m^{2}}$ and similarly for $E_{k^{\prime}}$. In Eq. (2.4) note that the $\psi_{\mathbf{k} ; S M_{S}, T}^{(-)}$'s satisfy incoming-wave boundary conditions, since these are the wave functions relevant for the process under consideration here, and that they have been expressed in terms of the $T$ matrix, defined as

$$
T_{M_{S}, M_{S}^{\prime}}^{S T}\left(\mathbf{k}, \mathbf{k}^{\prime}\right)=\left\langle\psi_{\mathbf{k} ; S M_{S}, T}^{(-)}(0)|v| \phi_{\mathbf{k}^{\prime} ; S M_{S}^{\prime}, T}(0)\right\rangle
$$

In Eq. (2.5), $\chi_{M_{S}, 0}^{S, T}$ denotes the $n p$ spin-isospin state $S M_{S}, T M_{T}=0$.

Bound or scattering wave functions in a frame moving with velocity $\mathbf{V}$ with respect to the rest frame are obtained from $[9,18]$

$$
\psi(\mathbf{p} ; \mathbf{V}) \equiv B(\mathbf{p}, \mathbf{V}) \psi\left(\mathbf{p}_{\|} / \gamma, \mathbf{p}_{\perp} ; 0\right)=\frac{1}{\sqrt{\gamma}}\left[1-\frac{\mathrm{i}}{4 m} \mathbf{V} \cdot\left(\boldsymbol{\sigma}_{1}-\boldsymbol{\sigma}_{2}\right) \times \mathbf{p}\right] \psi\left(\mathbf{p}_{\|} / \gamma, \mathbf{p}_{\perp} ; 0\right)
$$

where $\gamma=1 / \sqrt{1-V^{2}}$, and $\mathbf{p}_{\|}$and $\mathbf{p}_{\perp}$ denote the components of the momentum $\mathbf{p}$ parallel and perpendicular to $\mathbf{V}$, respectively. Only kinematical boost corrections are retained, in particular the spin-dependent ones associated with Thomas precession are only included to order $V^{2}$. The interaction-dependent corrections are ignored. However, it is interesting to note that Eqs. (2.4) and (2.7) suggest that, in order to boost the (fully interacting) scattering state, one only needs to know how to boost the free states.

\section{NUCLEAR ELECTROMAGNETIC CURRENT}

The electromagnetic current is taken as a sum of one- and two-body terms

$$
\mathbf{j}=\sum_{i=1,2} \mathbf{j}_{i}\left(\mathbf{p}_{i}^{\prime}, \mathbf{p}_{i}\right)+\mathbf{j}_{12}\left(\mathbf{p}_{1}^{\prime}, \mathbf{p}_{2}^{\prime}, \mathbf{p}_{1}, \mathbf{p}_{2}\right)
$$

The one-body term corresponds to the space part of the single-nucleon current $j_{i}^{\alpha}=\left(j_{i}^{0}, \mathbf{j}_{i}\right)$, with

$$
j_{i}^{\alpha}\left(\mathbf{p}_{i}^{\prime}, \mathbf{p}_{i}\right)=\bar{u}\left(\mathbf{p}_{i}^{\prime}\right)\left[F_{1, i}\left(Q^{2}\right) \gamma^{\alpha}+\frac{i}{2 m} F_{2, i}\left(Q^{2}\right) \sigma^{\alpha \beta} q_{\beta}\right] u\left(\mathbf{p}_{i}\right)
$$


where $u\left(\mathbf{p}_{i}\right)$ and $\bar{u}\left(\mathbf{p}_{i}^{\prime}\right)\left(\bar{u} \equiv u^{\dagger} \gamma^{0}\right)$ are the initial and final spinors of nucleon $i, \sigma^{\alpha \beta}=$ $(i / 2)\left[\gamma^{\alpha}, \gamma^{\beta}\right]$, and $F_{1, i}\left(Q^{2}\right)$ and $F_{2, i}\left(Q^{2}\right)$ denote respectively the nucleon's Dirac and Pauli form factors,

$$
F_{a, i}\left(Q^{2}\right) \equiv\left[F_{a}^{S}\left(Q^{2}\right)+F_{a}^{V}\left(Q^{2}\right) \tau_{i, z}\right] / 2, \quad a=1,2 .
$$

These form factors are normalized as $F_{1}^{S}(0)=F_{1}^{V}(0)=1$ and $F_{2}^{S}(0)=-0.12$ n.m. and $F_{2}^{V}(0)=3.706$ n.m. (in units of nuclear magnetons). The Höhler parameterization [19] of $F_{1}$ and $F_{2}$ is used in this work. The spinor $u$, or rather its adjoint, is given by

$$
u^{\dagger}(\mathbf{p})=\left(\frac{E+m}{2 E}\right)^{1 / 2}\left(\chi_{\sigma \tau}^{\dagger}, \chi_{\sigma \tau}^{\dagger} \frac{\boldsymbol{\sigma} \cdot \mathbf{p}}{E+m}\right)
$$

where $\mathbf{p}$ and $E=\sqrt{p^{2}+m^{2}}$ are the nucleon's momentum and energy, and $\chi_{\sigma \tau}$ is its (twocomponent) spin-isospin state. Note that $u^{\dagger} u=1$. Finally, the four-momentum transfer $q^{\mu}$, with $Q^{2}=-q^{\mu} q_{\mu}$, is taken in the Breit frame, in which the initial deuteron has momentum $-\mathbf{q} / 2$ and the final $n p$ pair has momentum $+\mathbf{q} / 2$, and is given by $q^{\mu}=(\omega, q \hat{\mathbf{z}})$ with $\omega=$ $E_{f}-E_{i}$, where $E_{i}=\sqrt{m_{d}^{2}+q^{2} / 4}\left(m_{d}\right.$ is the deuteron rest mass $)$ and $E_{f}=\sqrt{E_{k}^{2}+q^{2} / 4}\left(E_{k}\right.$ is the center-of-mass energy of the $n p$ pair, i.e. $E_{k}=2 \sqrt{k^{2}+m^{2}}$ ).

Assuming pseudovector $\pi$ - $N$ coupling, the two-body current associated with pion exchange is written as

$$
\mathbf{j}_{12}\left(\mathbf{p}_{1}^{\prime}, \mathbf{p}_{2}^{\prime}, \mathbf{p}_{1}, \mathbf{p}_{2}\right)=\mathbf{j}_{12}^{(a)}\left(\mathbf{p}_{1}^{\prime}, \mathbf{p}_{2}^{\prime}, \mathbf{p}_{1}, \mathbf{p}_{2}\right)+\mathbf{j}_{12}^{(b)}\left(\mathbf{p}_{1}^{\prime}, \mathbf{p}_{2}^{\prime}, \mathbf{p}_{1}, \mathbf{p}_{2}\right),
$$

where $\mathbf{j}_{12}^{(a)}$ is the current corresponding to the two seagull diagrams, and $\mathbf{j}_{12}^{(b)}$ is the current associated with the pion in flight diagram. They are given by

$$
\begin{aligned}
\mathbf{j}_{12}^{(a)}\left(\mathbf{p}_{1}^{\prime}, \mathbf{p}_{2}^{\prime}, \mathbf{p}_{1}, \mathbf{p}_{2}\right)= & i G_{E}^{V}\left(Q^{2}\right)\left(\boldsymbol{\tau}_{1} \times \boldsymbol{\tau}_{2}\right)_{z} \frac{f_{\pi N N}^{2}}{m_{\pi}^{2}} \frac{f_{\pi}^{2}\left(k_{2}\right)}{k_{2}^{2}-k_{2}^{02}+m_{\pi}^{2}} \\
& \bar{u}\left(\mathbf{p}_{1}^{\prime}\right) \gamma \gamma_{5} u\left(\mathbf{p}_{1}\right)\left[k_{2}^{\nu} \bar{u}\left(\mathbf{p}_{2}^{\prime}\right) \gamma_{\nu} \gamma_{5} u\left(\mathbf{p}_{2}\right)\right]+1 \rightleftharpoons 2, \\
\mathbf{j}_{12}^{(b)}\left(\mathbf{p}_{1}^{\prime}, \mathbf{p}_{2}^{\prime}, \mathbf{p}_{1}, \mathbf{p}_{2}\right)= & i G_{E}^{V}\left(Q^{2}\right)\left(\boldsymbol{\tau}_{1} \times \boldsymbol{\tau}_{2}\right)_{z} \frac{f_{\pi N N}^{2}}{m_{\pi}^{2}} \frac{f_{\pi}^{2}\left(k_{1}\right)}{k_{1}^{2}-k_{1}^{02}+m_{\pi}^{2}} \frac{f_{\pi}^{2}\left(k_{2}\right)}{k_{2}^{2}-k_{2}^{02}+m_{\pi}^{2}} \\
& \left(\mathbf{k}_{1}-\mathbf{k}_{2}\right)\left[k_{1}^{\nu} \bar{u}\left(\mathbf{p}_{1}^{\prime}\right) \gamma_{\nu} \gamma_{5} u\left(\mathbf{p}_{1}\right)\right]\left[k_{2}^{\rho} \bar{u}\left(\mathbf{p}_{2}^{\prime}\right) \gamma_{\rho} \gamma_{5} u\left(\mathbf{p}_{2}\right)\right],
\end{aligned}
$$

where the four-momentum $k_{i}^{\mu} \equiv\left(k_{i}^{0}, \mathbf{k}_{i}\right), i=1,2$, has $k_{i}^{0}=E_{i}^{\prime}-E_{i}$ and $\mathbf{k}_{i}=\mathbf{p}_{i}^{\prime}-\mathbf{p}_{i}$, and $f_{\pi N N}$ and $f_{\pi}\left(k_{i}\right)$ are respectively the pion-nucleon coupling constant and monopole form factor introduced previously. The fractional momenta $\mathbf{k}_{1}$ and $\mathbf{k}_{2}$ delivered to nucleons 1 and 2 add up to $\mathbf{q}$, that is $\mathbf{k}_{1}+\mathbf{k}_{2}=\mathbf{q}$. The nucleon isovector Sachs form factor $G_{E}^{V}\left(Q^{2}\right)$, 
related to $F_{1}^{V}\left(Q^{2}\right)$ and $F_{2}^{V}\left(Q^{2}\right)$ by $G_{E}^{V}\left(Q^{2}\right)=F_{1}^{V}\left(Q^{2}\right)-\left(Q^{2} / 4 m^{2}\right) F_{2}^{V}\left(Q^{2}\right)$, is used in the two-body currents. This choice is motivated by the following considerations. In the nonrelativistic limit, it is easy to show that the two-body pion-exchange current satisfies current conservation with the (non-relativistic) OPEP, obtained from Eq. (2.2) by setting $E=E^{\prime}=m$, if the same electromagnetic form factor is used in the charge operator and longitudinal component of the current. As shown in Appendix A, $G_{E}^{V}\left(Q^{2}\right)$ is used in the non-relativistic expression of the charge operator. Of course, the continuity equation places no restrictions on the electromagnetic form factors that may be used in the transverse components of the current. Ignoring this ambiguity, the choice $G_{E}^{V}\left(Q^{2}\right)$ satisfies the "minimal" requirement for current conservation. In the relativistic case, we choose to keep this same electromagnetic form factor in the two-body currents.

The full Lorentz structure of the one- and two-body currents is retained in the calculations reported here. The latter are listed, along with their respective non-relativistic limits, in Appendix A for completeness.

Finally, in earlier published work on the form factors and threshold electrodisintegration cross section of the deuteron $[14,17]$ and form factors of the $A=3-6$ nuclei, most recently $[20$, 21], the contributions associated with the boosts of the initial and final wave functions were neglected, and only terms up to order $(v / c)^{2}$ were included in the non-relativistic expansion of $j_{i}^{\alpha}$, namely the well known Darwin-Foldy and spin-orbit corrections to the charge operator $j_{i}^{0}$. Moreover, the two-body charge and current operators were taken to leading order.

\section{CALCULATION}

In the one-photon-exchange approximation, the differential cross section for deuteron electrodisintegration in the laboratory frame can be expressed as [22]

$$
\frac{d^{2} \sigma}{d \varepsilon^{\prime} d \Omega^{\prime}}=\sigma_{M}\left[W_{2}\left(Q^{2}, q_{\mu} P^{\mu}\right)+W_{1}\left(Q^{2}, q_{\mu} P^{\mu}\right) \tan ^{2}(\theta / 2)\right]
$$

where $\varepsilon^{\prime}$ and $\Omega^{\prime}$ are the final electron energy and solid angle, $\sigma_{M}$ is the Mott cross section, and the invariant response functions $W_{1}$ and $W_{2}$ depend on the square of the four momentum transfer, denoted as before by $Q^{2}$, and the Lorentz scalar $q^{\mu} P_{\mu}$, with $P_{\mu}$ being the four momentum of the deuteron in the initial state. At backward angles, the cross section above is dominated by $W_{1}$, i.e. transverse scattering. (Measurements of the deuteron threshold 
electrodisintegration have been performed at angles typically $\geq 155^{\circ}$, see Sec. V, for which $\tan ^{2}(\theta / 2)$ is $\geq 20$.) Hence, in the following, we will consider only the response $W_{1}$. In the Breit frame, defined in Sec. III, it can be written as

$$
W_{1}(q, \omega)=\sqrt{1+q^{2} /\left(2 m_{d}\right)^{2}} \sum_{S, T=0,1} R^{S T}(q, \omega)
$$

where the contribution from the individual spin-isospin states of the final $n p$ pair is given by

$$
R^{S T}(q, \omega)=\frac{1}{3} \sum_{M, M_{S}} \int \frac{d \mathbf{k}}{(2 \pi)^{3}} \frac{1}{2}\left|\mathbf{A}\left(q \hat{\mathbf{z}}, \mathbf{k} ; S, M_{S}, T, M\right)\right|^{2} \delta\left(E_{i}+\omega-E_{f}\right) .
$$

In the equation above, as in Sec. II, $M$ is the spin projection of the deuteron, and $S, M_{S}$, and $T$ specify the spin, spin projection, and isospin of the $n p$ scattering state, while in the energy-conserving $\delta$-function $E_{i}$ and $E_{f}$ are, respectively, the initial deuteron and final $n p$ pair Breit-frame energies, $E_{i}=\sqrt{q^{2} / 4+m_{d}^{2}}$ and $E_{f}=\sqrt{q^{2} / 4+E_{k}^{2}}$, with $E_{k}=2 \sqrt{k^{2}+m^{2}}$. Finally, the three-momentum transfer $\mathbf{q}$ is taken along the $\hat{\mathbf{z}}$ direction.

The amplitude $\mathbf{A}$ denotes the matrix elements of the transverse components (i.e., orthogonal to q) of the current operator, namely

$$
\mathbf{A}\left(q \hat{\mathbf{z}}, \mathbf{k} ; S, M_{S}, T, M\right)=\left\langle\psi_{\mathbf{k} ; S M_{S}, T}^{(-)}\left(\mathbf{V}_{f}\right)\left|\mathbf{j}_{\perp}(q \hat{\mathbf{z}})\right| \psi_{M}\left(\mathbf{V}_{i}\right)\right\rangle
$$

Here, $\psi_{M}\left(\mathbf{V}_{i}\right)$ and $\psi_{\mathbf{k} ; S M_{S}, T}^{(-)}\left(\mathbf{V}_{f}\right)$ are the deuteron and $n p$ scattering states boosted from the center-of-mass frame, where they are calculated in momentum space with the methods discussed in Sec. II, to the Breit frame, in which they have velocities given by, respectively, $\mathbf{V}_{i}=-(q / 2) \hat{\mathbf{z}} / E_{i}$ and $\mathbf{V}_{f}=+(q / 2) \hat{\mathbf{z}} / E_{f}$.

By inserting Eq. (2.4) into in Eq. (4.4), the amplitude A can be conveniently decomposed into the sum of two terms:

$$
\mathbf{A}=\mathbf{A}^{\mathrm{PW}}+\mathbf{A}^{\mathrm{FSI}}
$$

where

$$
\mathbf{A}^{\mathrm{PW}}\left(q \hat{\mathbf{z}}, \mathbf{k} ; S, M_{S}, T, M\right)=\left\langle\phi_{\mathbf{k} ; S M_{S}, T}\left(\mathbf{V}_{f}\right)\left|\mathbf{j}_{\perp}(q \hat{\mathbf{z}})\right| \psi_{M}\left(\mathbf{V}_{i}\right)\right\rangle
$$

and

$$
\mathbf{A}^{\mathrm{FSI}}\left(q \hat{\mathbf{z}}, \mathbf{k} ; S, M_{S}, T, M\right)=\sum_{M_{S}^{\prime}} \int \frac{d \mathbf{k}^{\prime}}{2(2 \pi)^{3}} \frac{T_{M_{S}, M_{S}^{\prime}}^{S T}\left(\mathbf{k}, \mathbf{k}^{\prime}\right)}{E_{k}-E_{k^{\prime}}+i \epsilon} \mathbf{A}^{\mathrm{PW}}\left(q \hat{\mathbf{z}}, \mathbf{k}^{\prime} ; S, M_{S}^{\prime}, T, M\right) .
$$

Thus, the amplitude $A^{\mathrm{PW}}$ corresponds to describing the final $n p$ states by plane waves (PW), while the amplitude $A^{\text {FSI }}$ takes into account interaction effects in these states. 
The electromagnetic current operator includes the one- and two-body terms discussed in the previous section. Details of the calculation of the amplitudes are reported in Appendix B.

\section{RESULTS AND CONCLUSIONS}

In this section we report the results obtained in the laboratory frame for the cross section of the deuteron threshold electrodisintegration at backward angles. The calculations were carried out with the relativistic (R) Hamiltonian of Sec. II, including the OPEP with offenergy-shell extension predicted by pseudovector coupling of pions to nucleons, i.e. with $\mu=+1$ in Eq. (2.2). This Hamiltonian was constructed to be phase-equivalent to the nonrelativistic (NR) Hamiltonian, based on the Argonne $v_{18}$ potential [17], using the methods developed in Ref. [11].

In Figs. 1 and 2 we show the deuteron and $n p{ }^{1} \mathrm{~S}_{0}$ wave functions, respectively, derived from the $\mathrm{R}$ and NR Hamiltonian models (the continuum wave function is calculated at a center-of-mass energy of $1.5 \mathrm{MeV}$ ). The deuteron $\mathrm{R} \mathrm{D}$-wave is larger than the $\mathrm{NR}$ at internucleon separations less than $1.5 \mathrm{fm}$, the corresponding D-state probabilities are $6.26 \%(\mathrm{R})$ and $5.76 \%(\mathrm{NR})$ - the difference has its origin in the local and non-local characters of the NR and R $(\mu+1)$ OPEP (for a discussion of this point, see Ref. [16]). However, the D- to S-state ratio and quadrupole moment, which are more sensitive to the wave functions in the asymptotic region, are respectively 0.0260 and $0.272 \mathrm{fm}^{2}$ in the $\mathrm{R}$ model, and 0.0250 and $0.270 \mathrm{fm}^{2}$ in the $\mathrm{NR}[16]$. In contrast, the $\mathrm{R}$ and $\mathrm{NR}^{1} \mathrm{~S}_{0}$ continuum wave functions hardly differ from each other, due to the vanishing of the tensor force in this channel.

In Fig. 3 we report the results for the electrodisintegration cross section obtained in the laboratory frame with the $\mathrm{R}$ (solid line) and NR (dashed line) Hamiltonian models and corresponding one-body currents, given respectively in Eqs. (A2) and (A4). The cross section obtained by ignoring the boost corrections for both the initial and final states in the $\mathrm{R}$ calculation - this is equivalent to setting $\mathbf{V}=0$ in Eq. (2.7) - is displayed by the dotted line, labeled RNB. All calculated cross sections include the contributions of $n p$ final states with total angular momentum up to $J=3$. These contributions are responsible for filling in the well-known nodal structure at $Q^{2} \simeq 12 \mathrm{fm}^{-2}$ in the cross section obtained with onebody currents by retaining only the ${ }^{1} \mathrm{~S}_{0}$ channel in the final state [12-15] (see Fig. 6 below). Finally, the inset of Fig. 3 shows the ratios of the $\mathrm{R}$ and RNB to the NR predictions. 


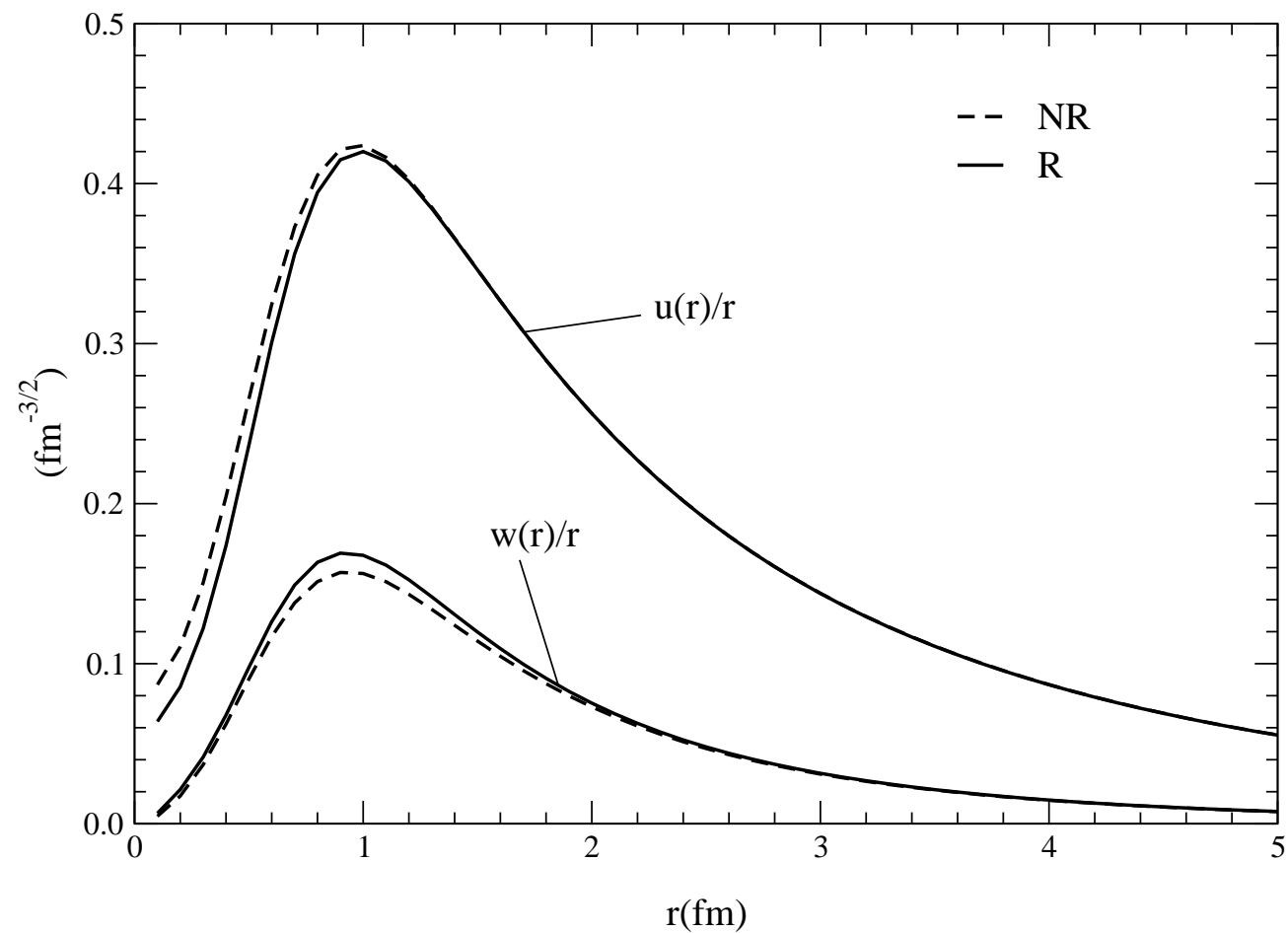

FIG. 1: The deuteron S- and D-state radial wave functions obtained with the R and NR Hamiltonian models.

The experimental data, labeled Bates, Saclay-81 and Saclay-85, are respectively from Refs. [25-27], and have been averaged over the interval $0-3 \mathrm{MeV}$ of the recoiling $n p$ pair center-of-mass energy; those labeled SLAC are from Ref. [28], and have been averaged over the interval 0-10 MeV. However, all theoretical curves in this figure, and following ones, have been calculated at a fixed center-of-mass energy of $1.5 \mathrm{MeV}$ and at an electron scattering angle $\theta=155^{\circ}$. The effect of the width of the energy interval above threshold of the final state, over which the cross section values are averaged, was studied in Ref. [14], and found to be very small. The electron scattering angles in the Saclay, Bates, and SLAC measurements were respectively $155^{\circ}, 160^{\circ}$, and $180^{\circ}$, but in fact the calculated cross section is weakly dependent on the specific value of the backward angle, since $\sigma_{M} \tan ^{2}(\theta / 2) \rightarrow \alpha^{2} /\left(4 \epsilon^{2}\right)$ as $\theta \rightarrow 180^{\circ}$ (here, $\alpha$ is fine structure constant and $\epsilon$ the initial electron energy).

The inset of Fig. 3 shows that the IA (R) and IA (NR) predictions differ significantly (i.e., more than $10 \%$ ) only for $Q^{2}>45 \mathrm{fm}^{-2}$. At lower momentum transfers, the IA (R) cross 


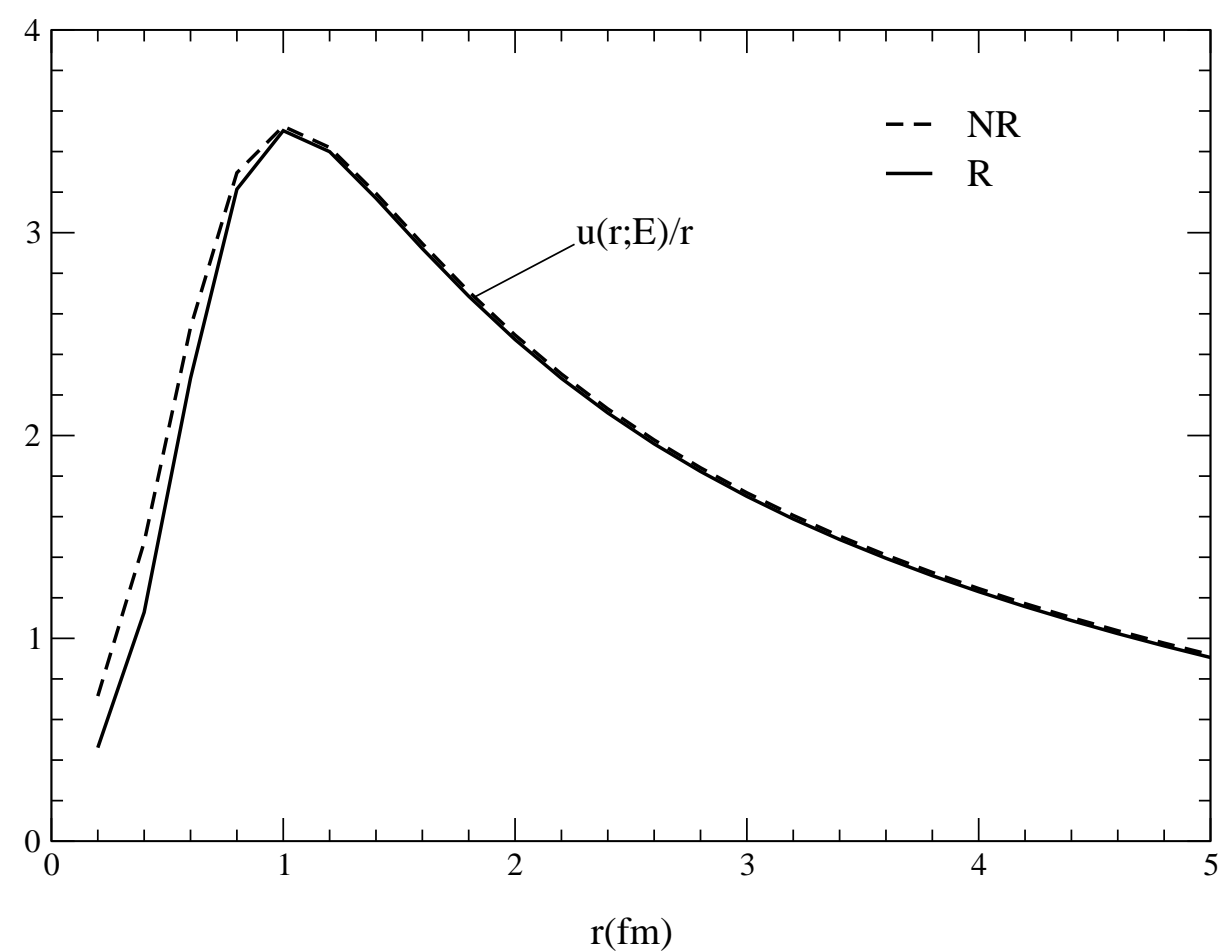

FIG. 2: The $n p{ }^{1} \mathrm{~S}_{0}$-state radial wave functions obtained with the $\mathrm{R}$ and NR Hamiltonian models at a center-of-mass energy of $1.5 \mathrm{MeV}$

section values are within $10 \%$ of the IA (NR). Comparison of the IA (R) and IA (RNB) results shows the effect of the boosts corrections in the initial deuteron and final $n p$ states. We have verified explicitly, by switching off the Thomas precession term in the boost operator of Eq. (2.7), that the dominant correction arises from the Lorentz contraction term (the resulting curve is essentially indistinguishable from that labeled IA (R); it is not shown to reduce clutter). Indeed, the IA (RNB) results can be approximately overlaid over the IA (R) results by multiplying the former by the factor $\left[1+Q^{2} /\left(16 m^{2}\right)\right]$, corresponding to the square of the Lorentz factor $\gamma \simeq \gamma_{i} \simeq \gamma_{f}=1 / \sqrt{1-V^{2}}$, where $V=|\mathbf{V}|=(q / 2) / \sqrt{4 m^{2}+q^{2} / 4}$, and the deuteron binding energy and $n p$ center-of-mass energy have been neglected. A similar effect was discussed in Ref. [9] in the context of a calculation of the deuteron electromagnetic form factors: it conforms with the naive expectation that the overlap between the initial and final states in configuration space is "squeezed" in the direction of motion (namely, along 


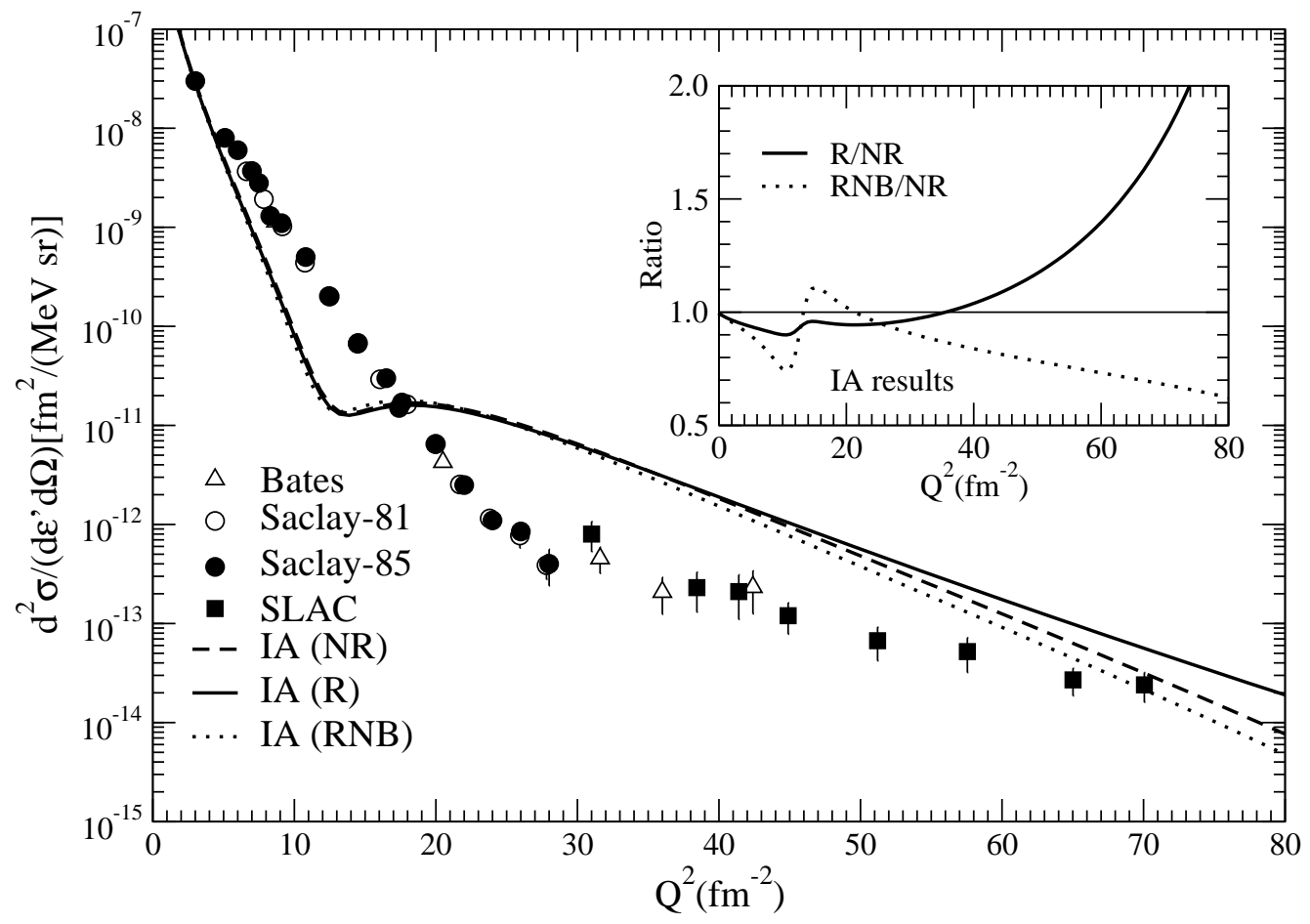

FIG. 3: The cross sections for deuteron threshold electrodisintegration at backward angles, obtained with the relativistic and non-relativistic Hamiltonian models and corresponding one-body currents [curves labeled IA (R) and IA (NR)] and by ignoring boost corrections in the relativistic calculation [curve labeled IA (RNB)], are compared with the experimental data from Refs. [25-28]. The inset displays the ratio of the IA (R) and IA (RNB) to the NR predictions.

q) by $\gamma$ or, equivelently, that its momentum space overlap is "pushed out" by $\gamma$.

In Fig. 4 we report the cross section results obtained by including, in addition to the single-nucleon current, the two-body current associated with pion exchange (curves labeled $\mathrm{IA}+\pi$ with $\mathrm{NR}, \mathrm{R}$, and RNB), while in the inset we display the ratios of $\mathrm{R}$ and RNB to NR predictions. For reference, we also show the IA $(R)$ cross sections presented in Fig. 3. The cross section values in the $\mathrm{R}$ calculation are significantly smaller than those in the NR in the momentum transfer range $Q^{2}=18-40 \mathrm{fm}^{-2}$. In fact, close inspection of Figs. 3-4 shows that the pion exchange contribution in the $\mathrm{R}$ calculation is larger than in the NR. In both of these calculations, this contribution is found to have the same sign, for $Q^{2}$ up to $\approx 12 \mathrm{fm}^{-2}$, as the one-body contribution. At larger $Q^{2}$ values, however, the latter changes sign, and the 


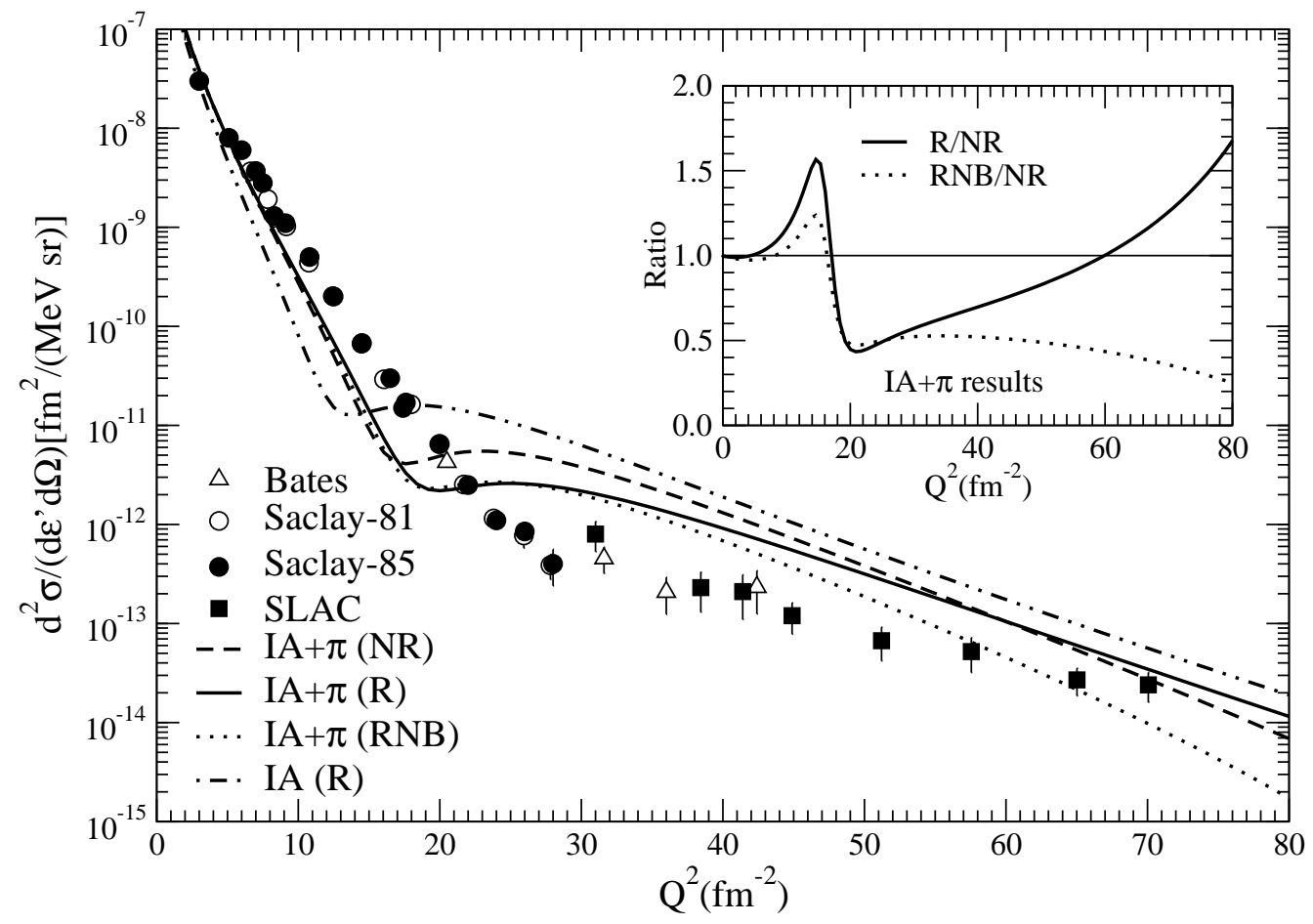

FIG. 4: Same as in Fig. 3, but with one-body and pion-exchange two-body currents. The cross sections obtained in the IA $(\mathrm{R})$ calculation are also shown.

resulting destructive interference between it and the two-body contribution is responsible for the suppression of the $\mathrm{R}$ cross section relative to the NR in this $Q^{2}$ region.

To investigate the mechanisms responsible for the suppression of the $\mathrm{R}$ relative to $\mathrm{NR}$ predictions for $Q^{2}$ in the range $18-40 \mathrm{fm}^{-2}$, we have carried out two different calculations, the results of which are displayed in Fig. 5. In the first, labeled IA $+\pi(\mathrm{NRW})$, we have replaced the NR expressions for the one- and two-body currents with the corresponding $\mathrm{R}$ ones, in order to isolate relativistic effects in the currents. Comparison between the IA $+\pi$ (NRW) and IA $+\pi(\mathrm{NR})$ curves shows that these effects reduce the cross section, for $Q^{2}>18$ $\mathrm{fm}^{-2}$.

In the second calculation, labeled IA $+\pi$ (NRC), we have used NR one- and two-body currents but $\mathrm{R}$ deuteron and $n p$ scattering wave functions without boost corrections - so this is the same as IA $+\pi(\mathrm{NR})$ calculation but for the replacement of the NR wave functions by the corresponding $\mathrm{R}$ ones - with the objective of isolating relativistic effects generated 
by the Hamiltonian. As in the previous case, we find that these reduce the cross section.

In Fig. 5 we also show the results of an $\mathrm{R}$ calculation in which the time components $\left(k_{i}^{0}\right)$ of the exchanged pion four-momenta in both the vertex operators and propagators of the two-body currents in Eqs. (A7)-(A8) are set to zero, curve labeled IA $+\pi(\mathrm{RK} 0)$. The latter essentially overlaps the IA $+\pi(\mathrm{R})$ curve. We have also verified by direct calculation that ignoring the retardation effects only in the pion propagators again hardly changes the IA $+\pi$ (R) predictions. Thus, the explicit energy dependence of the vertex operators implied by pseudovector coupling of pions to nucleons has a negligible effect.

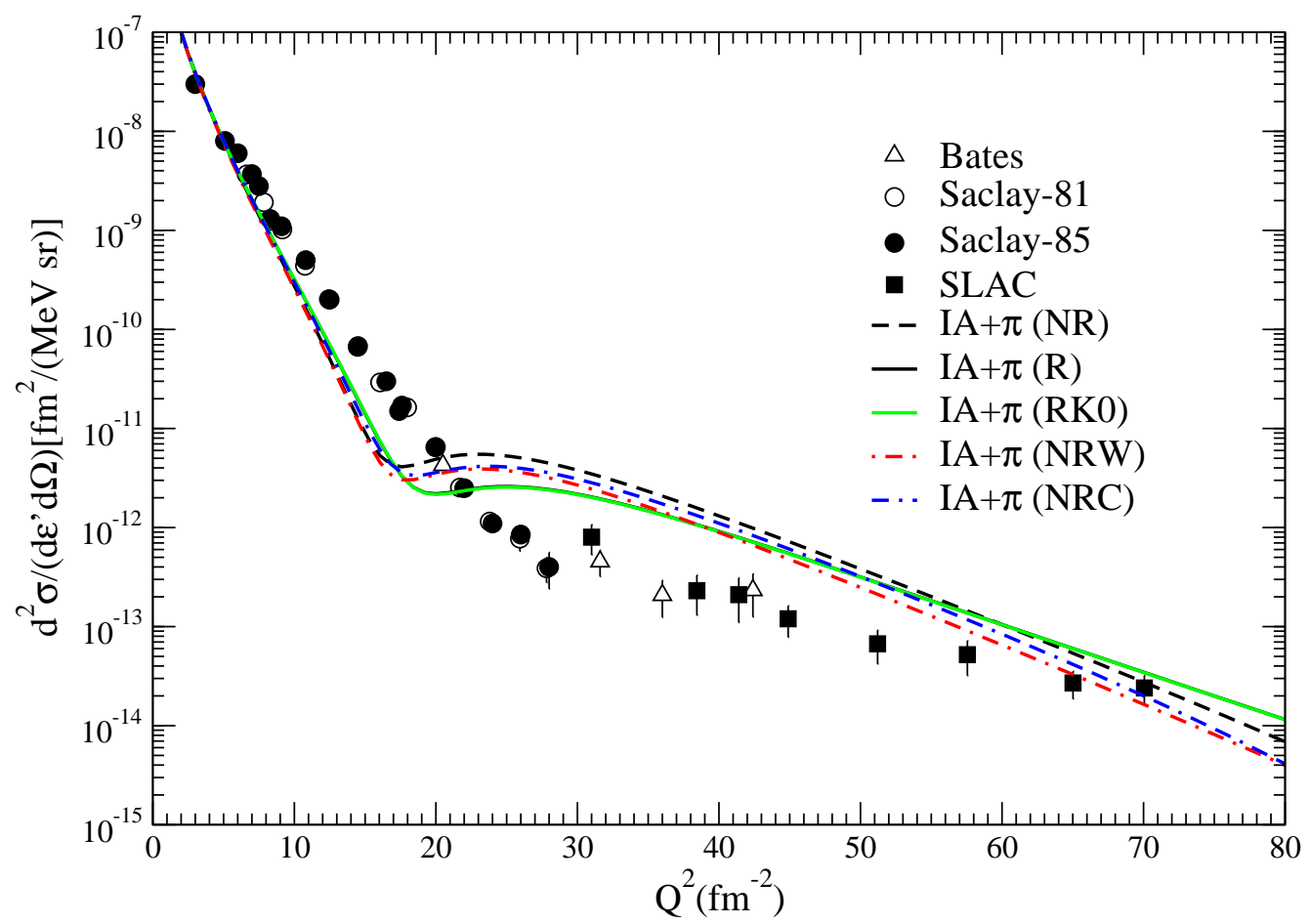

FIG. 5: The IA $+\pi(\mathrm{R})$ and $\mathrm{IA}+\pi(\mathrm{NR})$ predictions of Fig. 4 are compared with the results corresponding to different approximations, labeled respectively IA $+\pi(\mathrm{RK} 0), \mathrm{IA}+\pi(\mathrm{NRW})$, and $\mathrm{IA}+\pi$ (NRC). See text for discussion. The experimental data are from Refs. [25-28].

Figure 6 shows the results of $\mathrm{R}$ and NR calculations including only the ${ }^{1} \mathrm{~S}_{0}$ channel in the $n p$ final state compared both to data and the $\mathrm{R}$ results with the "complete" $n p$ state (all channels up to $J=3)$, i. e the curves labeled IA $(\mathrm{R})$ and $\mathrm{IA}+\pi(\mathrm{R})$ in Fig. 4. The nodes at $Q^{2} \approx 12 \mathrm{fm}^{-2}(\mathrm{IA})$ and 18 and $16 \mathrm{fm}^{-2}(\mathrm{IA}+\pi)$ in the $\mathrm{R}$ and $\mathrm{NR}$ predictions including only 


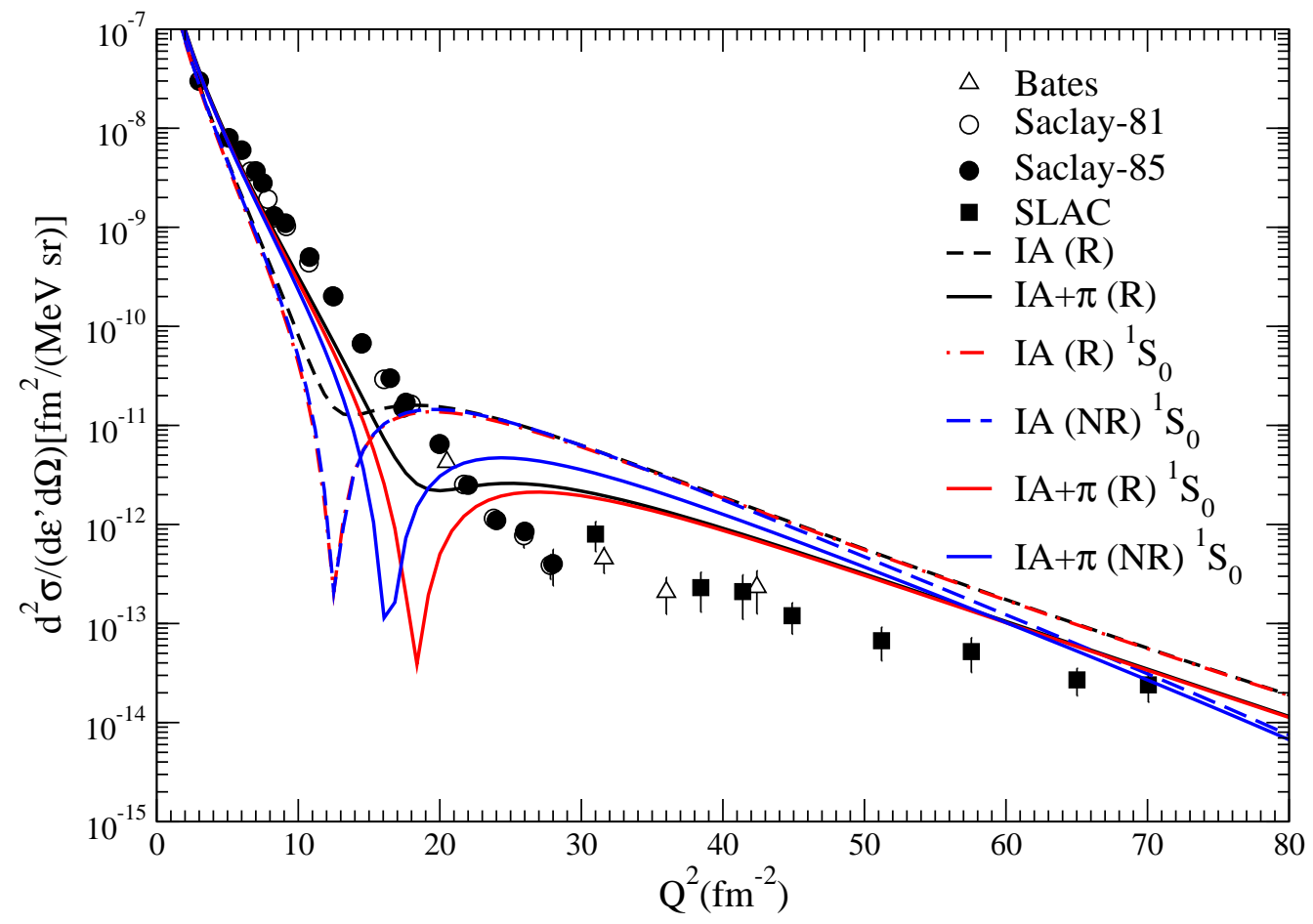

FIG. 6: The cross sections for deuteron threshold electrodisintegration at backward angles, obtained in the IA $(\mathrm{R})$ and $\mathrm{IA}+\pi(\mathrm{R})$ calculations, are compared with the results of calculations based on the NR and R Hamiltonian models and corresponding one- and two-body currents, but including only the ${ }^{1} \mathrm{~S}_{0}$ channel in the $n p$ final state. The experimental data are from Refs. [25-28].

the ${ }^{1} \mathrm{~S}_{0}$ channel are filled in by the contributions of higher partial waves in the complete calculations. The ${ }^{1} \mathrm{~S}_{0}$ IA $(\mathrm{R})$ and IA $(\mathrm{NR})$ cross sections are very close to each other, and thus confirm the conclusions of Ref. [15], in which the electrodisintegration cross sections was calculated within a relativistic approach based on light-front-form Hamiltonian dynamics, including only one-body currents.

To conclude, we find that relativistic effects in the calculations with only one-body currents become important (larger than 10\%) at momentum transfers $Q^{2}$ exceeding $40 \mathrm{fm}^{-2}$, and are due, for the most part, to boost corrections. However, when the pion-exchange current contributions are also taken into account, significant differences at lower $Q^{2}$ are obtained between the cross sections predicted within the $\mathrm{R}$ and NR models for the Hamiltonian and currents. The interplay between relativistic effects in the interactions and currents 
conspire to significantly reduce the cross section obtained in the $\mathrm{R}$ calculation in the $Q^{2}$ range $\simeq 18-40 \mathrm{fm}^{-2}$.

The cross section predictions based on both the $\mathrm{R}$ and $\mathrm{NR}$ models do not reproduce the experimental data at $Q^{2}>10 \mathrm{fm}^{-2}$, thus demonstrating the inadequacy of the present model for the electromagnetic current operator. This conclusion corroborates that of an earlier (NR) study [14], and suggests the need for including additonal (short-range) twobody currents.

Finally, in the present work we have not addressed the issue of current conservation within the $\mathrm{R}$ framework. Its discussion would require constructing the two-body charge operator associated with pion exchange, and studying the relation between the pion-exchange charge and current operators and the off-energy shell behavior of OPEP. This is beyond the scope of the present work.

\section{Acknowledgments}

The work of A.A. was partially supported by the Portuguese FCT under contract POCTI/FNU/49505/2002, while that of R.S. by the U.S. Department of Energy, Office of Nuclear Physics, under contract DE-AC05-06OR23177. One of the authors (A.A.) would like to thank the JLab Theory Group for the support and warm hospitality extended to her on several occasions. Finally, some of the calculations were made possible by grants of computing time from the National Energy Research Supercomputer Center.

\section{APPENDIX A: CURRENT OPERATOR EXPRESSIONS}

In this Appendix we list the expressions for the one-body and two-body pion-exchange current operators. The time (charge) and space (current) components of the one-body fourcurrent read, respectively:

$$
\begin{aligned}
& \rho_{i}\left(\mathbf{p}_{i}^{\prime}, \mathbf{p}_{i}\right)=N_{i}^{\prime} N_{i}\left[F_{1, i}\right.+F_{1, i} \frac{\mathbf{p}_{i}^{\prime} \cdot \mathbf{p}_{i}+i \boldsymbol{\sigma}_{i} \cdot \mathbf{p}_{i}^{\prime} \times \mathbf{p}_{i}}{\left(E_{i}^{\prime}+m\right)\left(E_{i}+m\right)} \\
&\left.+\frac{F_{2, i}}{2 m}\left(\frac{\mathbf{q} \cdot \mathbf{p}_{i}-i \boldsymbol{\sigma}_{i} \cdot \mathbf{p}_{i} \times \mathbf{q}}{E_{i}+m}-\frac{\mathbf{q} \cdot \mathbf{p}_{i}^{\prime}+i \boldsymbol{\sigma}_{i} \cdot \mathbf{p}_{i}^{\prime} \times \mathbf{q}}{E_{i}^{\prime}+m}\right)\right], \\
& \mathbf{j}_{i}\left(\mathbf{p}_{i}^{\prime}, \mathbf{p}_{i}\right)=N_{i}^{\prime} N_{i}\left\{F_{1, i}\left(\frac{\mathbf{p}_{i}-i \boldsymbol{\sigma}_{i} \times \mathbf{p}_{i}}{E_{i}+m}+\frac{\mathbf{p}_{i}^{\prime}+i \boldsymbol{\sigma}_{i} \times \mathbf{p}_{i}^{\prime}}{E_{i}^{\prime}+m}\right)\right.
\end{aligned}
$$




$$
\begin{aligned}
& +\frac{F_{2, i}}{2 m} \omega\left(\frac{\mathbf{p}_{i}-i \boldsymbol{\sigma}_{i} \times \mathbf{p}_{i}}{E_{i}+m}-\frac{\mathbf{p}_{i}^{\prime}+i \boldsymbol{\sigma}_{i} \times \mathbf{p}_{i}^{\prime}}{E_{i}^{\prime}+m}\right) \\
& -i \frac{F_{2, i}}{2 m} \mathbf{q} \times \boldsymbol{\sigma}_{i}\left[1+\frac{\mathbf{p}_{i}^{\prime} \cdot \mathbf{p}_{i}}{\left(E_{i}^{\prime}+m\right)\left(E_{i}+m\right)}\right]+i \frac{F_{2, i}}{2 m} \frac{\boldsymbol{\sigma}_{i} \cdot \mathbf{p}_{i}^{\prime} \mathbf{q} \times \mathbf{p}_{i}+\boldsymbol{\sigma}_{i} \cdot \mathbf{p}_{i} \mathbf{q} \times \mathbf{p}_{i}^{\prime}}{\left(E_{i}^{\prime}+m\right)\left(E_{i}+m\right)} \\
& \left.+\frac{F_{2, i}}{2 m} \frac{\mathbf{q} \cdot \mathbf{p}_{i} \mathbf{p}_{i}^{\prime}-\mathbf{q} \cdot \mathbf{p}_{i}^{\prime} \mathbf{p}_{i}}{\left(E_{i}^{\prime}+m\right)\left(E_{i}+m\right)}\right\},
\end{aligned}
$$

where the spinor-normalization factor $N_{i}=\sqrt{\left(E_{i}+m\right) /\left(2 E_{i}\right)}$ and similarly for $N_{i}^{\prime}, \boldsymbol{\sigma}_{i}$ is the Pauli spin operator of nucleon $i$, and the initial and final nucleon spin-isospin states $\chi_{\sigma_{i}^{\prime} \tau_{i}^{\prime}}$ and $\chi_{\sigma_{i} \tau_{i}}$ are not explicitly shown. The initial and final momenta are denoted respectively as $\mathbf{p}_{i}$ and $\mathbf{p}_{i}^{\prime}$, while the energy and three-momentum transfers $\omega$ and $\mathbf{q}$ are taken in the Breit frame, defined in Sec. III after Eq. (3.4). The non-relativistic limits to order $(p / m)^{2}$ included are written as

$$
\begin{aligned}
& \rho_{i}^{\mathrm{NR}}\left(\mathbf{p}_{i}^{\prime}, \mathbf{p}_{i}\right)=\frac{G_{E, i}}{\sqrt{1+Q^{2} /(4 m)^{2}}}+i \frac{2 G_{M, i}-G_{E, i}}{4 m^{2}} \boldsymbol{\sigma}_{i} \cdot \mathbf{p}_{i}^{\prime} \times \mathbf{p}_{i} \\
& \mathbf{j}_{i}^{\mathrm{NR}}\left(\mathbf{p}_{i}^{\prime}, \mathbf{p}_{i}\right)=\frac{G_{E, i}}{2 m}\left(\mathbf{p}_{i}+\mathbf{p}_{i}^{\prime}\right)+i \frac{G_{M, i}}{2 m} \boldsymbol{\sigma}_{i} \times \mathbf{q}
\end{aligned}
$$

where $Q^{2}=-q^{\mu} q_{\mu}$ and the Sachs nucleon form factors, defined as

$$
\begin{aligned}
G_{E, i} & =F_{1, i}-\frac{Q^{2}}{4 m^{2}} F_{2, i} \\
G_{M, i} & =F_{1, i}+F_{2, i}
\end{aligned}
$$

have been introduced in Eqs. (A3)-(A4). The two-body pion-exchange currents are given by

$$
\begin{aligned}
& \mathbf{j}_{12}^{(a)}\left(\mathbf{p}_{1}^{\prime}, \mathbf{p}_{2}^{\prime}, \mathbf{p}_{1}, \mathbf{p}_{2}\right)= i N_{1}^{\prime} N_{2}^{\prime} N_{1} N_{2} G_{E}^{V}\left(Q^{2}\right) \frac{f_{\pi N N}^{2}}{m_{\pi}^{2}} \frac{f_{\pi}^{2}\left(k_{2}\right)}{k_{2}^{2}-k_{2}^{02}+m_{\pi}^{2}}\left(\boldsymbol{\tau}_{1} \times \boldsymbol{\tau}_{2}\right)_{z} \\
& {\left[\boldsymbol{\sigma}_{1}+\frac{\left(\boldsymbol{\sigma}_{1} \cdot \mathbf{p}_{1}^{\prime}\right) \boldsymbol{\sigma}_{1}\left(\boldsymbol{\sigma}_{1} \cdot \mathbf{p}_{1}\right)}{\left(E_{1}^{\prime}+m\right)\left(E_{1}+m\right)}\right]\left\{k_{2}^{0}\left(\frac{\boldsymbol{\sigma}_{2} \cdot \mathbf{p}_{2}^{\prime}}{E_{2}^{\prime}+m}+\frac{\boldsymbol{\sigma}_{2} \cdot \mathbf{p}_{2}}{E_{2}+m}\right)\right.} \\
&- {\left.\left[\boldsymbol{\sigma}_{2} \cdot \mathbf{k}_{2}+\frac{\left(\boldsymbol{\sigma}_{2} \cdot \mathbf{p}_{2}^{\prime}\right) \boldsymbol{\sigma}_{2} \cdot \mathbf{k}_{2}\left(\boldsymbol{\sigma}_{2} \cdot \mathbf{p}_{2}\right)}{\left(E_{2}^{\prime}+m\right)\left(E_{2}+m\right)}\right]\right\}+1 \rightleftharpoons 2, } \\
& \mathbf{j}_{12}^{(b)}\left(\mathbf{p}_{1}^{\prime}, \mathbf{p}_{2}^{\prime}, \mathbf{p}_{1}, \mathbf{p}_{2}\right)=i N_{1}^{\prime} N_{2}^{\prime} N_{1} N_{2} G_{E}^{V}\left(Q^{2}\right) \frac{f_{\pi N N}^{2}}{m_{\pi}^{2}} \frac{f_{\pi}^{2}\left(k_{1}\right)}{k_{1}^{2}-k_{1}^{02}+m_{\pi}^{2}} \frac{f_{\pi}^{2}\left(k_{2}\right)}{k_{2}^{2}-k_{2}^{02}+m_{\pi}^{2}}\left(\boldsymbol{\tau}_{1} \times \boldsymbol{\tau}_{2}\right)_{z} \\
&\left(\mathbf{k}_{1}-\mathbf{k}_{2}\right)\left\{k_{1}^{0}\left(\frac{\boldsymbol{\sigma}_{1} \cdot \mathbf{p}_{1}^{\prime}}{E_{1}^{\prime}+m}+\frac{\boldsymbol{\sigma}_{1} \cdot \mathbf{p}_{1}}{E_{1}+m}\right)-\left[\boldsymbol{\sigma}_{1} \cdot \mathbf{k}_{1}+\frac{\left(\boldsymbol{\sigma}_{1} \cdot \mathbf{p}_{1}^{\prime}\right) \boldsymbol{\sigma}_{1} \cdot \mathbf{k}_{1}\left(\boldsymbol{\sigma}_{1} \cdot \mathbf{p}_{1}\right)}{\left(E_{1}^{\prime}+m\right)\left(E_{1}+m\right)}\right]\right\} \\
&\left\{k_{2}^{0}\left(\frac{\boldsymbol{\sigma}_{2} \cdot \mathbf{p}_{2}^{\prime}}{E_{2}^{\prime}+m}+\frac{\boldsymbol{\sigma}_{2} \cdot \mathbf{p}_{2}}{E_{2}+m}\right)-\left[\boldsymbol{\sigma}_{2} \cdot \mathbf{k}_{2}+\frac{\left(\boldsymbol{\sigma}_{2} \cdot \mathbf{p}_{2}^{\prime}\right) \boldsymbol{\sigma}_{2} \cdot \mathbf{k}_{2}\left(\boldsymbol{\sigma}_{2} \cdot \mathbf{p}_{2}\right)}{\left(E_{2}^{\prime}+m\right)\left(E_{2}+m\right)}\right]\right\},(\mathrm{A} 8)
\end{aligned}
$$


where $k_{i}^{0}=E_{i}^{\prime}-E_{i}$ and $\mathbf{k}_{i}=\mathbf{p}_{i}^{\prime}-\mathbf{p}_{i}$, and the product of three Pauli matrices can be further reduced via the identity

$$
\left(\boldsymbol{\sigma}_{i} \cdot \mathbf{p}_{i}^{\prime}\right) \boldsymbol{\sigma}_{i}\left(\boldsymbol{\sigma}_{i} \cdot \mathbf{p}_{i}\right)=\mathbf{p}_{i}^{\prime}\left(\boldsymbol{\sigma}_{i} \cdot \mathbf{p}_{i}\right)+\mathbf{p}_{i}\left(\boldsymbol{\sigma}_{i} \cdot \mathbf{p}_{i}^{\prime}\right)-\boldsymbol{\sigma}_{i}\left(\mathbf{p}_{i}^{\prime} \cdot \mathbf{p}_{i}\right)+i\left(\mathbf{p}_{i}^{\prime} \times \mathbf{p}_{i}\right) .
$$

To leading order in $(p / m)^{2}$, the non-relativistic limits in Eqs. (A7)-(A8) sum up to

$$
\begin{aligned}
\mathbf{j}_{12}\left(\mathbf{k}_{1}, \mathbf{k}_{2}\right) & =-i G_{E}^{V}\left(Q^{2}\right) \frac{f_{\pi N N}^{2}}{m_{\pi}^{2}}\left(\boldsymbol{\tau}_{1} \times \boldsymbol{\tau}_{2}\right)_{z}\left\{\left[\boldsymbol{\sigma}_{1}\left(\boldsymbol{\sigma}_{2} \cdot \mathbf{k}_{2}\right) \frac{f_{\pi}^{2}\left(k_{2}\right)}{k_{2}^{2}+m_{\pi}^{2}}-1 \rightleftharpoons 2\right]\right. \\
& \left.-\left(\mathbf{k}_{1}-\mathbf{k}_{2}\right)\left(\boldsymbol{\sigma}_{1} \cdot \mathbf{k}_{1}\right)\left(\boldsymbol{\sigma}_{2} \cdot \mathbf{k}_{2}\right) \frac{f_{\pi}^{2}\left(k_{1}\right)}{k_{1}^{2}+m_{\pi}^{2}} \frac{f_{\pi}^{2}\left(k_{2}\right)}{k_{2}^{2}+m_{\pi}^{2}}\right\} .
\end{aligned}
$$

\section{APPENDIX B: CALCULATION OF AMPLITUDES}

In this Appendix we outline the method used to compute the matrix elements of the one-body and two-body current operators in Eqs. (4.6)-(4.7). The calculation is carried out in the Breit frame, in which the initial deuteron and final $n p$ pair (with center-of-mass energy $E_{k}=2 \sqrt{k^{2}+m^{2}}$ ) have velocities given respectively by

$$
\mathbf{V}_{i}=-\frac{\mathbf{q}}{2 \sqrt{m_{d}^{2}+q^{2} / 4}}, \quad \mathbf{V}_{f}=+\frac{\mathbf{q}}{2 \sqrt{E_{k}^{2}+q^{2} / 4}} .
$$

Here the momentum transfer $\mathbf{q}$ is taken along the $\hat{\mathbf{z}}$-axis. We also define $\gamma_{i}=1 / \sqrt{1-V_{i}^{2}}$ and similarly for $\gamma_{f}$.

The computer codes implementing the formalism discussed below have been successfully tested by comparing, in a model calculation which ignored boost corrections and kept only the leading terms in the non-relativistic expansions of the one- and two-body currents, the present results with those obtained [14] with an earlier, configuration-space version of the code.

\section{One-body amplitude}

Following Eq. (4.5), we decompose the one-body amplitude into PW and FSI amplitudes. The PW amplitude is written as

$$
\mathbf{A}_{1-\text { body }}^{\mathrm{PW}}\left(\mathbf{q} ; \mathbf{k}, S, M_{S}, T, M\right)=2 \int \frac{d \mathbf{p}}{(2 \pi)^{3}} \phi_{\mathbf{k} ; S M_{S}, T}^{\dagger}\left(\mathbf{p}+\mathbf{q} / 2 ; \mathbf{V}_{f}\right) \mathbf{j}_{\perp, 1}\left(\mathbf{p}_{1}^{\prime}, \mathbf{p}_{1}\right) \psi_{M}\left(\mathbf{p} ; \mathbf{V}_{i}\right),
$$


where $\mathbf{p}$ is the relative momentum, $\mathbf{p}_{1}^{\prime}=3 \mathbf{q} / 4+\mathbf{p}$ and $\mathbf{p}_{1}=-\mathbf{q} / 4+\mathbf{p}$, the factor of 2 in front of the integral takes into account the identical contribution coming from the current of nucleon $2, \mathbf{j}_{\perp, 2}$, and finally $\psi_{M}\left(\mathbf{p} ; \mathbf{V}_{i}\right)$ and $\phi_{\mathbf{k} ; S M_{S}, T}\left(\mathbf{p}+\mathbf{q} / 2 ; \mathbf{V}_{f}\right)$ are the momentum-space deuteron and free $n p$ wave functions, boosted to the Breit frame. Making the change of integration variables $\left[\left(\mathbf{p}_{\|}+\mathbf{q} / 2\right) / \gamma_{f}, \mathbf{p}_{\perp}\right] \rightarrow\left(\mathbf{p}_{\|}, \mathbf{p}_{\perp}\right)$, where $\mathbf{p}_{\|}$and $\mathbf{p}_{\perp}$ refer respectively to the components of $\mathbf{p}$ parallel and perpendicular to $\mathbf{q}$, leads to the following expression for $\mathbf{A}_{1-\text { body }}^{\mathrm{PW}}$

$$
\begin{array}{r}
\mathbf{A}_{1-\text { body }}^{\mathrm{PW}}\left(\mathbf{q} ; \mathbf{k}, S, M_{S}, T, M\right)=2 \gamma_{f} \int \frac{d \mathbf{p}}{(2 \pi)^{3}} \phi_{\mathbf{k} ; S M_{S}, T}^{\dagger}(\mathbf{p} ; 0) B^{\dagger}\left(\mathbf{p}, \mathbf{V}_{f}\right) \mathbf{j}_{\perp, 1}\left(\overline{\mathbf{p}}_{1}^{\prime}, \overline{\mathbf{p}}_{1}\right) \\
B\left(\mathbf{p}, \mathbf{V}_{i}\right) \psi_{M}\left[\left(\gamma_{f} \mathbf{p}_{\|}-\mathbf{q} / 2\right) / \gamma_{i}, \mathbf{p}_{\perp}\right]
\end{array}
$$

where $\overline{\mathbf{p}}_{1}^{\prime}=\gamma_{f} \mathbf{p}_{\|}+\mathbf{q} / 4+\mathbf{p}_{\perp}$ and $\overline{\mathbf{p}}_{1}=\gamma_{f} \mathbf{p}_{\|}-3 \mathbf{q} / 4+\mathbf{p}_{\perp}$, and the boost operators $B\left(\mathbf{p}, \mathbf{V}_{i}\right)$ and $B\left(\mathbf{p}, \mathbf{V}_{f}\right)$ can be read off from Eq. (2.7) (note that under the change of variables above, the Thomas precession term remains unchanged, since both $\mathbf{V}_{i}$ and $\mathbf{V}_{f}$ are along $\mathbf{q}$ ).

It is convenient to expand the free $n p$ wave function in partial waves [23]:

$$
\phi_{\mathbf{k} ; S M_{S}, T}(\mathbf{p} ; 0)=\sqrt{2}(2 \pi)^{3} \frac{\delta(k-p)}{k p} \sum_{L J M_{J}} \epsilon_{L S T}\left[Z_{L S M_{S}}^{J M_{J}}(\hat{\mathbf{k}})\right]^{*} \mathcal{Y}_{L S J}^{M_{J}}(\hat{\mathbf{p}}) \chi_{0}^{T}
$$

where $\epsilon_{L S T}=\left[1-(-)^{L+S+T}\right] / 2$,

$$
Z_{L S M_{S}}^{J M_{J}}(\hat{\mathbf{k}})=\sum_{M_{L}}\left\langle L M_{L}, S M_{S} \mid J M_{J}\right\rangle Y_{L M_{L}}(\hat{\mathbf{k}})
$$

and $\mathcal{Y}_{L S J}^{M_{J}}$ are standard spin-angle functions. Inserting this expansion in Eq. (B3) gives

$$
\mathbf{A}_{1-\text { body }}^{\mathrm{PW}}\left(\mathbf{q} ; \mathbf{k}, S, M_{S}, T, M\right)=\sum_{L J M_{J}} \epsilon_{L S T} Z_{L S M_{S}}^{J M_{J}}(\hat{\mathbf{k}}) J_{L J M_{J} ; M}^{S T}(\mathbf{q}, k)
$$

where

$$
\begin{array}{r}
J_{L J M_{J} ; M}^{S T}(\mathbf{q}, k)=2 \sqrt{2} \gamma_{f} \int d \Omega_{\mathbf{p}} \chi_{0}^{T \dagger} \mathcal{Y}_{L S J}^{M_{J} \dagger}(\hat{\mathbf{p}}) B^{\dagger}\left(\mathbf{p}, \mathbf{V}_{f}\right) \mathbf{j}_{\perp, 1}\left(\overline{\mathbf{p}}_{1}^{\prime}, \overline{\mathbf{p}}_{1}\right) \\
B\left(\mathbf{p}, \mathbf{V}_{i}\right) \psi_{M}\left[\left(\gamma_{f} \mathbf{p}_{\|}-\mathbf{q} / 2\right) / \gamma_{i}, \mathbf{p}_{\perp}\right]
\end{array}
$$

and the magnitude of the relative momentum is fixed by the $\delta$-function in Eq. (B4) to be $|\mathbf{p}|=k$ (note that $\mathbf{p}$ enters in the arguments of the boost and current operators and deuteron wave function). For an assigned set of quantum numbers $L J M_{J} ; M$ and $S T$, the function $J(\mathbf{q}, k)$ is calculated efficiently by standard Gaussian integrations over the $\hat{\mathbf{p}}$-directions. 
In order to evaluate the FSI amplitude, we first introduce in Eq. (4.7) the partial wave expansions for $\mathbf{A}_{1-\text { body }}^{\mathrm{PW}}$, Eq. (B6), and for the T-matrix [23],

$$
T_{M_{S}, M_{S}^{\prime}}^{S T}\left(\mathbf{k}, \mathbf{k}^{\prime}\right)=2(4 \pi)^{2} \sum_{J M_{J} L L^{\prime}} i^{L^{\prime}-L} \epsilon_{L S T} \epsilon_{L^{\prime} S T} Z_{L S M_{S}}^{J M_{J}}(\hat{\mathbf{k}})\left[Z_{L^{\prime} S M_{S}^{\prime}}^{J M_{J}}\left(\hat{\mathbf{k}}^{\prime}\right)\right]^{*} T_{L L^{\prime}}^{S T J}\left(k, k^{\prime}\right)
$$

and then carry out the integrations over the $\hat{\mathbf{k}}^{\prime}$ solid angle to obtain

$$
\mathbf{A}_{1-\text { body }}^{\mathrm{FSI}}\left(\mathbf{q} ; \mathbf{k}, S, M_{S}, T, M\right)=\sum_{L J M_{J}} \epsilon_{L S T} Z_{L S M_{S}}^{J M_{J}}(\hat{\mathbf{k}}) J_{L J M_{J} ; M}^{S T}(\mathbf{q}, k ; \mathrm{FSI})
$$

where we have defined

$$
J_{L J M_{J} ; M}^{S T}(\mathbf{q}, k ; \mathrm{FSI})=\sum_{L^{\prime}} i^{L^{\prime}-L} \epsilon_{L^{\prime} S T}\left[\frac{2}{\pi} \int_{0}^{\infty} d k^{\prime} k^{\prime 2} \frac{T_{L L^{\prime}}^{S T J}\left(k, k^{\prime}\right)}{E_{k}-E_{k^{\prime}}+i \epsilon} J_{L^{\prime} J M_{J} ; M}^{S T}\left(\mathbf{q}, k^{\prime}\right)\right] .
$$

In deriving the equations above, use has been made of the following relation:

$$
\sum_{M_{S}} \int d \Omega_{\mathbf{k}}\left[Z_{L S M_{S}}^{J M_{J}}(\hat{\mathbf{k}})\right]^{*} Z_{L^{\prime} S M_{S}}^{J^{\prime} M_{J}^{\prime}}(\hat{\mathbf{k}})=\delta_{J J^{\prime}} \delta_{M_{J} M_{J}^{\prime}} \delta_{L L^{\prime}}
$$

while a standard subtraction technique [24] is employed to perform the principal value integration implicit in Eq. (B10).

In the partial wave expansions of the amplitudes, fully converged results for $\mathbf{A}_{1-\text { body }}^{\mathrm{PW}}$ and $\mathbf{A}_{1-\text { body }}^{\mathrm{FSI}}$ are obtained, at the low center-of-mass energy of the final $m p$ pair of interest here (1.5 MeV), when all contributions with total angular momentum $J \leq 3$ are retained in the sum over channels.

\section{Two-body amplitude}

In this case, after rescaling the $\mathbf{p}^{\prime}$ relative momentum as $\left(\mathbf{p}_{\|}^{\prime} / \gamma_{f}, \mathbf{p}_{\perp}^{\prime}\right) \rightarrow\left(\mathbf{p}_{\|}^{\prime}, \mathbf{p}_{\perp}^{\prime}\right)$ in the integral, the PW amplitude reads

$$
\begin{aligned}
\mathbf{A}_{2-\text { body }}^{\mathrm{PW}}\left(\mathbf{q} ; \mathbf{k}, S, M_{S}, T, M\right)= & \gamma_{f} \int \frac{d \mathbf{p}^{\prime}}{(2 \pi)^{3}} \frac{d \mathbf{p}}{(2 \pi)^{3}} \phi_{\mathbf{k} ; S M_{S}, T}^{\dagger}\left(\mathbf{p}^{\prime} ; 0\right) B^{\dagger}\left(\mathbf{p}^{\prime}, \mathbf{V}_{f}\right) \\
& \mathbf{j}_{\perp, 12}\left(\overline{\mathbf{p}}_{1}^{\prime}, \overline{\mathbf{p}}_{2}^{\prime}, \overline{\mathbf{p}}_{1}, \overline{\mathbf{p}}_{2}\right) B\left(\mathbf{p}, \mathbf{V}_{i}\right) \psi_{M}\left(\mathbf{p}_{\|} / \gamma_{i}, \mathbf{p}_{\perp}\right)
\end{aligned}
$$

where $\overline{\mathbf{p}}_{1}^{\prime}=\mathbf{q} / 4+\gamma_{f} \mathbf{p}_{\|}^{\prime}+\mathbf{p}_{\perp}^{\prime}, \overline{\mathbf{p}}_{2}^{\prime}=\mathbf{q} / 4-\gamma_{f} \mathbf{p}_{\|}^{\prime}-\mathbf{p}_{\perp}^{\prime}$ and $\overline{\mathbf{p}}_{1}=-\mathbf{q} / 4+\mathbf{p}, \overline{\mathbf{p}}_{2}=-\mathbf{q} / 4-\mathbf{p}$. Rather than expanding the free $n p$ state in partial waves, we carry out the $\mathbf{p}^{\prime}$ integration by inserting into the equation above the plane waves of Eq. (2.5), and obtain

$$
\begin{aligned}
\mathbf{A}_{2-\text { body }}^{\mathrm{PW}}\left(\mathbf{q} ; \mathbf{k}, S, M_{S}, T, M\right)= & \sqrt{2} \gamma_{f} \int \frac{d \mathbf{p}}{(2 \pi)^{3}} \chi_{M_{S}, 0}^{S, T \dagger} B^{\dagger}\left(\mathbf{k}, \mathbf{V}_{f}\right) \\
& \mathbf{j}_{\perp, 12}\left(\overline{\mathbf{p}}_{1}^{\prime}, \overline{\mathbf{p}}_{2}^{\prime}, \overline{\mathbf{p}}_{1}, \overline{\mathbf{p}}_{2}\right) B\left(\mathbf{p}, \mathbf{V}_{i}\right) \psi_{M}\left(\mathbf{p}_{\|} / \gamma_{i}, \mathbf{p}_{\perp}\right)
\end{aligned}
$$


where in the momenta $\overline{\mathbf{p}}_{1}^{\prime}$ and $\overline{\mathbf{p}}_{2}^{\prime}$ the parallel and perpendicular components of the relative momentum $\mathbf{p}^{\prime}$ are replaced by those corresponding to $\mathbf{k}$. The three-dimensional integrations in Eq. (B13) are done by Gaussian quadratures.

The amplitudes $\mathbf{A}_{2-\text { body }}^{\mathrm{FSI}}$ are calculated from Eq. (4.7) by direct integration over $\mathbf{k}^{\prime}$. To this end, we first reconstruct, from the channel solutions $T_{L L^{\prime}}^{S T J}\left(k, k^{\prime}\right)$, the full $T$-matrix in Eq. (B8), by including contributions with total angular momentum up to $J=3$, and then use cubic-spline techniques to interpolate the $\mathbf{A}_{2-\text { body }}^{\mathrm{PW}}$, previously tabulated on a sufficiently coarse grid, at the $\mathbf{k}^{\prime}$ values relevant for integration.

[1] F. Gross, Phys. Rev. 186, 1448 (1969); Phys. Rev. D 10, 223 (1974); Phys. Rev. C 26, $2203(1982)$.

[2] R. Blankenbecler and R. Sugar, Phys. Rev. 142, 1051 (1966).

[3] B.D. Keister and W.N. Polyzou, Adv. Nucl. Phys. 20, 225 (1991).

[4] R. Gilman and F. Gross, J. Phys. G 28, R37 (2002).

[5] J.W. Van Orden, N. Devine, and F. Gross, Phys. Rev. Lett. 75, 4369 (1995).

[6] A. Stadler and F. Gross, Phys. Rev. Lett. 78, 26 (1997).

[7] F.M. Lev, E. Pace, and G. Salmè, Phys. Rev. C 62, 064004 (2000).

[8] T.W. Allen, W.H. Klink, and W.N. Polyzou, Phys. Rev. C 63, 034002 (2001).

[9] R. Schiavilla and V.R. Pandharipande, Phys. Rev. C 65, 064009 (2002).

[10] J.L. Forest, V.R. Pandharipande and A. Arriaga, Phys. Rev. C60, 014002 (1999).

[11] J. Carlson, V.R. Pandharipande, and R. Schiavilla, Phys. Rev. C 47, 484 (1993).

[12] J. Hockert, D.O. Riska, M. Gari, and A. Huffman, Nucl. Phys. A 217, 14 (1973).

[13] W. Leidemann, K.-M. Schmitt, and H. Arenhövel, Phys. Rev. C 42, 826 (1990).

[14] R. Schiavilla and D.O. Riska, Phys. Rev. C 43, 437 (1991).

[15] B.D. Keister, Phys. Rev. C 37, 1765 (1988).

[16] J.L. Forest, Phys. Rev. C 61, 034007 (2000).

[17] R.B. Wiringa, V.G.J. Stoks, and R. Schiavilla, Phys. Rev. C 51, 38 (1995).

[18] J.L. Friar, Ann. Phys. (N.Y.) 104, 380 (1977).

[19] G. Höhler, Nucl. Phys. B 114, 505 (1976).

[20] L.E. Marcucci, D.O. Riska, and R. Schiavilla, Phys. Rev. C 58, 3069 (1998). 
[21] R.B. Wiringa and R. Schiavilla, Phys. Rev. Lett. 81, 4317 (1998).

[22] T. deForest and J.D. Walecka, Adv. Phys. 15, 1 (1966).

[23] R. Schiavilla, J. Carlson, and M. Paris, Phys. Rev. C 70, 044007 (2004).

[24] W. Glöckle, The Quantum Mechanical Few-Body Problem (Springer-Verlag, Berlin, 1983).

[25] W.M. Schmitt et al., Phys. Rev. C 56, 1687 (1997).

[26] M. Bernheim et al., Phys. Rev. Lett. 46, 402 (1981).

[27] S. Auffret et al., Phys. Rev. Lett. 55, 1362 (1985).

[28] M. Frodyma et al., Phys. Rev. C 47, 1599 (1993). 